\title{
Adaptation-level theory and the free recall of mixed-frequency lists
}

\author{
DAVID C. RUBIN \\ Duke University, Durham, North Carolina 27706 \\ and \\ STEPHEN CORBETT \\ University of Wisconsin, Madison, Wisconsin 53706
}

\begin{abstract}
Subjects learned a list containing both high-frequency (common) and low-frequency (rare) words after learning five lists of either high- or low-frequency words. As predicted by adaptationlevel theory, preexposure to lists at one frequency made words at that same frequency more difficult to learn relative to words at other frequencies.
\end{abstract}

The psychological value of a stimulus is usually defined in absolute terms in studies of memory (e.g., in terms of its meaningfulness, imagery, or frequency). Definition in terms of the stimulus' context, however, often proves useful. In particular, stimuli near the extremes of a range tend to be learned easier or recalled better than those in the middle. In paired associate learning, such U-shaped functions have been found when the stimulus or response members varied along such dimensions as location, brightness, loudness, size, the value of integers, and the meaning of adjectives covering the range from ugly to beautiful (see Bower, 1971, for a review). Less relevant research has been performed using free recall, but, in addition to the frequency dimension to be considered here, the physical size of nonsense syllables has been shown to produce a Ushaped function (Nachmias, 1958). This paper examines the word-frequency dimension using the most comprehensive of the relativistic views, adaptation-level theory (Helson, 1964).

Lists of high-frequency (common) words are better recalled than are lists of low-frequency (rare) words (Deese, 1960; Hall, 1954). However, this is not always the case for lists of mixed-frequency words. In an early study, Peters (1936) demonstrated that in lists of mixed-frequency words high- and low-frequency words are recalled more often than are medium-frequency words. Nachmias (1958) has replicated this finding, and Rubin (1974) has found that the effect may hold even in prose. Peters also noted that the terms high, low, and medium are not absolute but, rather, are defined by the list as a whole. That is, whether Peters chose a list

Harry Helson was a Distinguished Visiting Professor at Lawrence University in the fall semester of 1974. This note grew out of that visit. Reprints are available from David C. Rubin, Psychology Department, Duke University, Durham, North Carolina 27706. covering the most common 2,500 words, or the most common 5,000 words, or the least common 7,000 words from Thorndike's (1921) list of 10,000 words, he always obtained a U-shaped function for amount recalled vs. frequency (cf. Bower, 1971; Nachmias, 1958).

Adaptation-level theory (Helson, 1964) holds that the effectiveness of a stimulus is determined by its position relative to subject's adaptation level, rather than in any absolute terms. In the case of mixed-frequency lists, the adaptation level would be equal to a weighted geometric mean of the stimuli in the list and all past stimuli. By assuming that the distinctiveness of a stimulus is monotonically related to its distance from the adaptation level and by assuming a relationship between distinctiveness and learning, predictions can be made about learning (Bower, 1971; Capehart, Tempone, \& Herbert, 1969; Helson, 1964; Murdock, 1960).

On a qualitative level, Peters' (1936) findings offer strong support for the role of adaptation-level theory in list learning. First, words with frequencies away from the geometric mean of the stimuli of the list are recalled better than are words near the geometric mean. Second, this U-shaped function holds for lists with very different geometric means, ruling out the possibility that the effect is due to some absolute property of the words rather than to relations among them.

A reanalysis of a more modern study also offers support for a U-shaped function in mixed-frequency lists. Christian, Bickley, Tarka, and Clayton (1978) had subjects recall lists of words using a standard list learning paradigm in order to obtain percentage recalled values for 899 of Paivio, Yuille, and Madigan's (1968) 925 nouns. These 899 words, which are distributed approximately uniformly in log frequency, were rank ordered by frequency of occurrence (Kuðera \& Francis, 1967) and then combined into five groups of 180 , $180,180,180$, and 179 words each. The five groups had 
geometric mean frequencies of $1,4,12,33$, and 127 . The mean percentages recalled for these groups were $40 \%, 38 \%, 39 \%, 38 \%$, and $44 \%$. If the words were considered to be independent observations, the recall values would represent a small but significant departure from a flat curve $[\mathrm{F}(4,894)=5.19, \mathrm{p}<.001]$ and a U-shaped planned comparison $(3,-2,-2,-2,3)$ would be significant $[\mathrm{t}(894)=3.68, \mathrm{p}<.001]$. Peters $(1936)$ and others (Duncan, 1974; Gregg, 1976; May \& Tryk, 1970) note that subjects recall more low-frequency than highfrequency words in mixed-frequency lists. The reanalysis of Christian et al. (1978) as well as other studies (Balota \& Neely, 1980; Smith, Glenberg, \& Bjork, 1978) does not support this finding, indicating that the higher recall of low-frequency words in mixed lists may depend in part on yet-to-be-controlled factors (Gregg, 1976; Paivio \& Madigan, 1970).

Adaptation-level theory predicts effects not only within lists but also between lists. No matter where a subject's adaptation level to word frequency is prior to an experiment, the learning of extremely high-frequency word lists should raise that subject's adaptation level and, therefore, should make low-frequency words more distinctive. Likewise, a subject who studies and recalls extremely low-frequency words should find highfrequency words more distinctive. Thus, if subjects are presented a test list of mixed high- and low-frequency words after several lists of high-frequency words, their recalls should differ from subjects who have studied and recalled several lists of low-frequency words.

\section{METHOD}

\section{Subjects}

Forty-eight undergraduate volunteers took part in the experiment.

\section{Procedure}

The subjects' instructions were as follows: "I am going to show you six lists of 16 words each. The words will appear on the screen one at a time. After each list you will have two minutes in which to write down as many words as you can remember." Subjects were tested individually. The words were presented one every $4 \mathrm{sec}$. The subject was in one room, and the experimenter and the slide projector were in another.

The first five lists were anchor lists. For half the subjects, these consisted of all high-frequency words, and for the other half, they consisted of all low-frequency words. The sixth list was the target list, which consisted of half high- and half lowfrequency words. Verbatim recall was required.

\section{Materials}

Kučera and Francis (1967) was the source of all 208 nouns used in the experiment. The low-frequency words were randomly sampled from words having a frequency of $5 /$ million. The high-frequency words, which were selected from frequencies ranging from 228 to $847 /$ million, had a geometric mean of 336. From each of the 104 high- and low-frequency words, 24 words were selected to be in the target lists. The remaining words were used in the five anchor lists.

For the target lists, plurals were not used and word length was equated across frequency in an attempt to limit two of the many differences between high- and low-frequency words (Landauer \& Streeter, 1973; Rubin, 1980). Three target lists of 16 words each were formed by alternating 8 high-and 8 lowfrequency words, thus exhausting the pool of 24 high- and lowfrequency target words. After one subject in the high anchor condition and one subject in the low anchor condition saw each of those lists (i.e., after six subjects), the target lists were shuffled and new target lists were formed.

\section{RESULTS}

The high-frequency anchor lists were remembered better than the low-frequency anchor lists $(57 \%$ vs. $43 \%)$, replicating this standard finding $[\mathrm{F}(1,46)=21.93$, $\mathrm{p}<.001]$. For the mixed-frequency target lists, in the high anchor conditions the low-frequency words were recalled better than the high-frequency words $(69 \%$ vs. $52 \%$ ), whereas in the low anchor condition $55 \%$ of both the high- and low-frequency words were recalled correctly. The interaction predicted by adaptation-level theory was significant $[F(1,46)=9.18, p<.005]$, and the main effect of frequency in mixed-frequency lists is in agreement with most studies $[\mathrm{F}(1,46)=9.18$, $\mathrm{p}<.005]$. The main effect of anchor type $[\mathrm{F}(1,46)=$ 1.23] was not significant.

\section{DISCUSSION}

Adaptation-level theory (Helson, 1964) can account for three effects in the learning of mixed-frequency lists: (1) that medium-frequency words are not recalled as well as high-or lowfrequency words, (2) that medium, high, and low are defined in terms of the list used and not in terms of absolute frequency, and (3) that exposure to lists at one frequency makes words at that frequency more difficult to recall relative to words at other frequencies. Many questions, however, remain unanswered. What is the actual psychological dimension along which adaptation and distinctiveness occur? (Any variable correlated with frequency could be involved.) Could the adaptation-level explanation be accounted for by other explanations (e.g., proactive inhibition), and could such explanations be distinguished? Rather than pursue these and other questions here, the simple point will be made that adaptation is a far-reaching phenomenon often overlooked at the current time in memory and cognition.

Evidence abounds that judgments, perceptions, and even learning are often related to a person's current state rather than to an absolute value (Appley, 1971; Helson, 1964). This view tends to be ignored, however, because it can make psychological explanations more complex. Word frequency, or any continuum used in psychology, is much easier to conceptualize if it is absolute rather than relative to the past experience. Theories that make predictions completely in terms of the current state of the world are simpler than theories that postulate a memory to record the recent past.

Over the range of most continua studied in psychology, using absolute magnitudes rather than magnitudes measured relative to earlier experience provides a good approximation to the data. Our practice of averaging results from many random orders both acknowledges the effects of recent past experience and helps reduce them to apparent noise (Staddon, King, \& Lockhead, 1980). While adaptation-level effects may be present if large ranges and set orders are used, they may not appear when more limited ranges of normally distributed, randomly presented stimuli are used (e.g, Rubin, 1980). This situation has both advantages and disadvantages for psycho- 
logical theorizing. On the positive side, adaptation-level effects are often small enough to be combined into the error term without obscuring other regularities. On the negative side, adaptationlevel effects that should affect the basic way we view psychological processes are often forgotten. This paper is a reminder of their existence.

\section{REFERENCES}

Appley, M. H. (Ed.). Adaptation-level theory. New York: Academic Press, 1971.

Balota, D. A., \& NeEly, J. H. Text-expectancy and wordfrequency effects in recall and recognition. Journal of Experimental Psychology: Human Learning and Memory, 1980, 6, 576-587.

Bower, G. H. Adaptation-level coding of stimuli and serial position effects. In M. H. Appley (Ed.), Adaptation-level theory. New York: Academic Press, 1971.

Capehart, J., Tempone, V. J., \& Herbert, J. A theory of stimulus equivalence. Psychological Review, 1969, 76, $405-418$.

Christian, J., Bickley, W., Tarka, M., \& Clayton, K. Measures of free recall of 900 English nouns: Correlations with imagery, concreteness, meaningfulness, and frequency. Memory \& Cognition, 1978, 6, 379-390.

DEESE, J. Frequency of usage and number of words in free recall: The role of association. Psychological Reports, 1960, 7, 337 344.

Duncan, C. P. Retrieval of low-frequency words from mixed lists. Bulletin of the Psychonomic Society, 1974, 4, 137-138.

GREGG, V. Word frequency, recognition, and recall. In J. Brown (Ed.), Recall and recognition. New York: Wiley, 1976.

Hall, J. F. Learning as a function of word-frequency. American Journal of Psychology, 1954, 67, 138-140.

Helson, H. Adaptation-level theory: An experimental and systematic approach to behavior. New York: Harper \& Row, 1964.

KuCERA, H., \& Francis, W. N. Computational analysis of present-day American English. Providence, R.I: Brown University Press, 1967.
Landauer, T. K., \& Streeter, L. A. Structural differences between common and rare words: Failure of equivalence assumptions for theories of word recognition. Journal of Verbal Learning and Verbal Behavior, 1973, 12, 119-131.

MAY, R. B., \& TrYK, H. E. Word sequence, word frequency, and free recall. Canadian Journal of Psychology, 1970, 24, 299-304.

Murdock, B. B., JR. The distinctiveness of stimuli. Psychological Review, 1960, 67, 16-31.

Nachmias, J. The effect of stimulus-heterogeneity on free recall. American Journal of Psychology, 1958, 71, 578-582.

Paivio, A., \& Madigan, S. A. Noun imagery and frequency in paired-associate and free-recall learning. Canadian Journal of Psychology, 1970, 24, 353-361.

Paivio, A., Yuille, J. C., \& Madigan, S. A. Concreteness, imagery, and meaningfulness values for 925 nouns. Journal of Experimental Psychology Monograph Supplement, 1968, 76(1, Pt. 2).

Peters, H. N. The relationship between familiarity of words and their memory value. American Journal of Psychology, 1936, 48, 572-584.

RuBIN, D. C. Remembering prose. Unpublished doctoral dissertation, Harvard University, 1974.

RuBin, D. C. 51 properties of 125 words: A unit analysis of verbal behavior. Journal of Verbal Learning and Verbal Behavior, 1980, 19, 736-755.

Smith, S. M., Glenberg, A., \& Bjork, R. A. Environmental context and human memory. Memory \& Cognition, 1978, 6, 342-353.

Staddon, J. E. R., King, M., \& Lockhead, G. R. On sequential effects in absolute judgment experiments. Journal of Experimental Psychology: Human Perception and Performance, 1980, 6, 290-301.

ThORNDIKE, E. L. The teacher's word book. New York: Teachers College, Columbia University Press, 1921.

(Received for publication May 7, 1982.) 